\title{
In situ cleaning/passivation of surfaces for contact technology on III-V materials
}

\author{
Philippe Rodriguez*, Laura Toselli*, Élodie Ghegin*†, Fabrice Nemouchi*, Névine Rochat* and Eugénie Martinez* \\ *Univ. Grenoble Alpes, F-38000 Grenoble, France \\ CEA, LETI, MINATEC Campus, F-38054 Grenoble, France. \\ Email: philippe.rodriguez@cea.fr \\ ${ }^{\dagger}$ STMicroelectronics, 850 rue Jean Monnet, BP 16, 38926 Crolles, France
}

\begin{abstract}
In this work we introduce the use of physical plasmas (e.g. Ar- and He-based plasmas) in order to study the in situ cleaning (prior to metal deposition) of InGaAs layers dedicated to the realisation of self-aligned contacts. For the characterisation of cleaning efficiency, we performed surface analyses like $X$ ray photoelectron spectroscopy and Fourier transform infrared spectroscopy in attenuated total reflection mode. The first results described in this work are encouraging. We have found efficient processes for removing totally or partially III-V native oxides.
\end{abstract}

\section{INTRODUCTION - CONTEXT}

The contact technology for III-V compounds has been developed since the pioneering article of Dale and Turner in 1963 [1] and the obtaining of simple Sn-based Ohmic contacts on gallium arsenide. The interest for contacting III-V materials has been growing since disruptive channel materials (including $\mathrm{Ge}$ and III-V materials) have been proposed for boosting the CMOS performance [2].

In recent years, for integration and scaling reasons, efforts have been made to develop non-gold Ohmic contacts and to reduce the contact dimensions from micrometric to nanometric scale. For advanced CMOS, the aim is to obtain a contact technology more cost-effective and compatible with the $\mathrm{Si}$ CMOS technology. In this way, we observed a transition from non-self aligned technology to a self-aligned process [3], [4]. To date, the most studied materials are the Ni-InGaAs and Co-InGaAs alloys [5], [6].

In order to propose a suitable contact technology on III$\mathrm{V}$ materials, whatever the technology used (non-self aligned, self aligned, 3D...), cleaning/passivation technology is highly desirable for removing particles, organic materials, metallic impurities and native oxides. The classical salicide process (eg. NiSi on $\mathrm{Si}$ or $\mathrm{SiGe} 30 \%$ ) involves a two-step procedure where a wet HF treatment is coupled with an in situ hot $\mathrm{NF}_{3} / \mathrm{NH}_{3}$ remote plasma exposure. Queue time related surface contamination caused by using only wet chemical cleaning is thus eliminated. For III-V contact technology, we strongly think that a similar strategy has to be developed. If wet chemical cleanings are generally proposed for realising InGaAs nMOSFET, in situ cleanings have not been extensively studied. For deeply scaled transistors, parasitic resistances between the S/D regions and the metal contact have to be reduced as much as possible. Then, a very cleaned and native oxide free surface must be obtained before metal deposition. Thus, the absence of a dry in situ cleaning before contact metallisation could be a real issue and have strong effects on the contact microstructural and electrical characteristics.
In this work, we propose a first study using a $\mathrm{Si}$ CMOS technology compatible equipment in order to investigate the in situ cleaning/passivation of III-V surfaces. In this paper, we focused on results obtained with physical plasmas (e.g. Arand He-based plasmas).

\section{EXPERIMENTAL DETAILS}

All experiments described in this work were carried out in a $300 \mathrm{~mm}$ Applied Materials Endura® platform usually dedicated to silicide process. Apart from the metal RF PVD chambers, this tool is equipped with various pre-clean chambers where one can realise sample heating, hot $\mathrm{NF}_{3} / \mathrm{NH}_{3}$ remote plasma exposure and argon- or helium-based etching plasmas. The aim of this work is to use the pre-clean chambers usually assigned to Si-based wafers and to adapt the plasma chemistry and process parameters for the cleaning of III-V surfaces.

Because large III-V wafers are not available, we used small pieces of III-V materials on dedicated holders in order to process the samples in the $300 \mathrm{~mm}$ platform. In this work, we focused on results obtained for InGaAs layers grown on InP or Si substrates.

The passivation/cleaning efficiency and impact on III-V surfaces were studied by using surface analyses like X-ray photoelectron spectroscopy (XPS) and Fourier transform infrared (FTIR) spectroscopy in attenuated total reflection (ATR) mode. For FTIR-ATR characterisations, the data were collected using a Ge prism pressed against the sample. A Bruker IFS 55 FTIR spectrometer probed the sample via a $\mathrm{P}$ polarized infrared beam through the prism at an incident angle of $65^{\circ}$, ensuring total reflexion on the Ge prism dioptre. XPS spectra were collected in a PHI VersaProbe II Scanning XPS Microprobe system using a monochromatic $\mathrm{Al} \mathrm{K}_{\alpha}$ X-ray source. In $3 \mathrm{~d}$, As 3d, Ga 3d and In 4d spectra were collected with a constant pass energy of $23.5 \mathrm{eV}$ leading to an overall energy resolution of $0.6 \mathrm{eV}$; curve fitting was done by CasaXPS 2.3.16 software.

\section{RESULTS}

The processes based on Ar- or He-based etching plasmas are usually dedicated to the etching of tens of nanometres of silicon oxide. Thus, they have been adapted to obtain plasmas compatible with the thickness of III-V native oxides (typically ranging from 1 to 5 nanometres). One orientation was to reduce the plasma power in order to limit the impact on the III-V surface in terms of defects and roughness. 
The figure 1 exhibits the normalized FTIR-ATR spectra for an InGaAs reference layer (i.e. native oxides without any treatments) and for InGaAs layers after Ar- and He-based plasma processes.

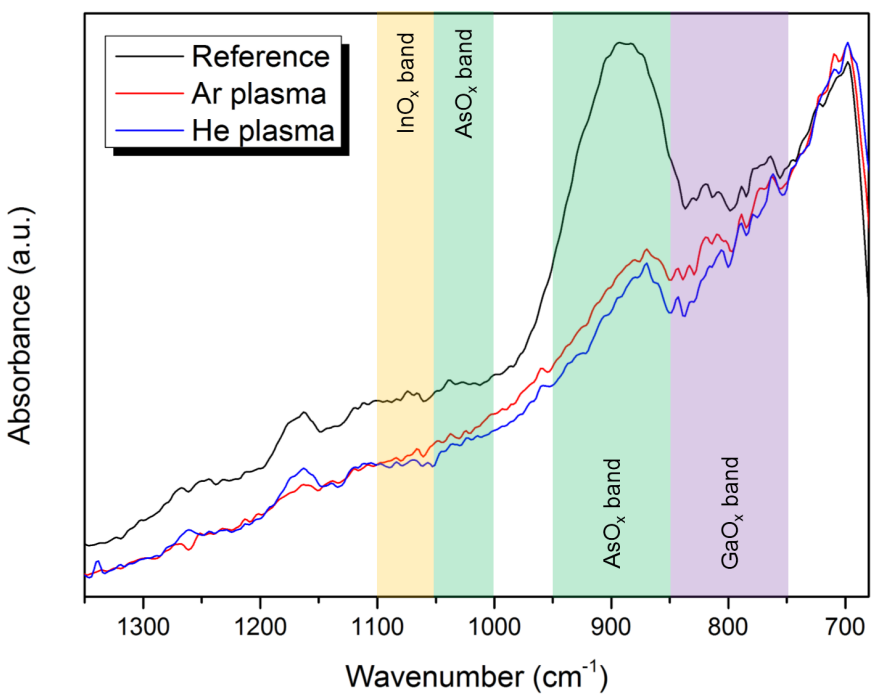

Fig. 1. Normalized Fourier transform infrared spectroscopy spectra in attenuated total reflection mode for InGaAs layers treated with Ar plasma (red line), He plasma (blue line) and without cleaning/passivation treatment (black line)

Based on literature data [7], [8], the absorption regions of III-V oxides are highlighted on figure 1 . The bands observed in the $1100-1150 \mathrm{~cm}^{-1}$ and $1150-1200 \mathrm{~cm}^{-1}$ regions are not attributed to III-V oxides but might be related to carbonaceous pollution from Ge prism or samples.

We clearly observe differences between the spectrum obtained for an uncleaned sample (black spectrum) and the spectra obtained for the plasma treated ones (red and blue spectra). Especially, the large absorption band of $\mathrm{AsO}_{\mathrm{x}}$ in the region of $850-950 \mathrm{~cm}^{-1}$ is drastically reduced after surface treatment. The impact of plasma treatments on $\mathrm{Ga}$ and In oxides is also seen but it is less obvious.

The figure 2 shows the normalized FTIR-ATR spectra for InGaAs layers treated with $\mathrm{He}$ (red line), $\mathrm{He} / \mathrm{H}_{2}$ 20/1 (blue line), $\mathrm{He} / \mathrm{H}_{2}$ 10/1 (magenta line) plasmas and without cleaning/passivation treatment (black line).

From the results depicted on figure 2, some tendencies can be drawn. First, the introduction of a slight percent of hydrogen in He-based plasma seems to modify the III-V oxides cleaning efficiency. The effect of hydrogen is visible for the large absorption band of $\mathrm{AsO}_{\mathrm{x}}$ in the $850-950 \mathrm{~cm}^{-1}$ and 1000 $-1050 \mathrm{~cm}^{-1}$ regions. Regarding, the shape and the intensity of the corresponding bands, $\mathrm{AsO}_{\mathrm{x}}$ removal appears to be inversely proportional to the $\mathrm{H}_{2}$ plasma content. It is less pronounced, but a similar tendency can be observed for $\mathrm{GaOx}$ bands. For In oxides, no significant effect can be detected.

Even if this technique is not often reported in literature, in this work, we demonstrated that FTIR in ATR mode is a fast and adapted technique for studying III-V material surface and discriminating efficient from non-efficient plasma processes.

For the "ATR-efficient treatments" we performed XPS

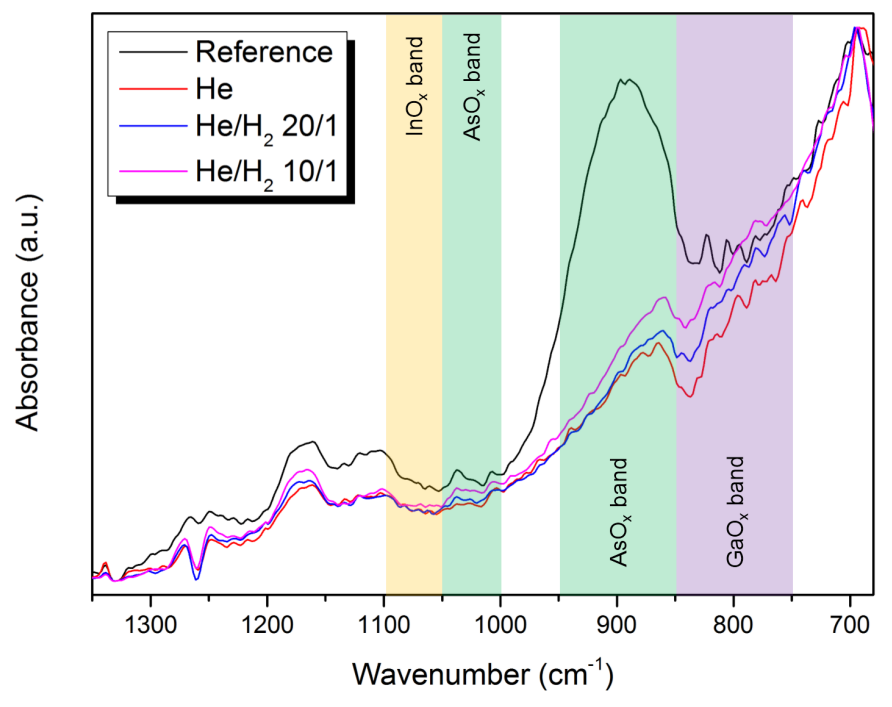

Fig. 2. Normalized Fourier transform infrared spectroscopy spectra in attenuated total reflection mode for InGaAs layers treated with $\mathrm{He}$ (red line), $\mathrm{He} / \mathrm{H}_{2}$ 20/1 (blue line), $\mathrm{He} / \mathrm{H}_{2}$ 10/1 (magenta line) plasmas and without cleaning/passivation treatment (black line).

analyses. An example of the obtained results is shown on figure 3 . The XPS results clearly evidence that plasma treatments were efficient for reducing the percentage of III-V oxides. It is obvious for As oxides where the $\mathrm{As}_{2} \mathrm{O}_{5}$ component is no more detected and the $\mathrm{As}_{2} \mathrm{O}_{3}$ is drastically reduced for passivated samples. For In and Ga oxides, the proportion of oxide components are significantly reduced compared to the reference sample. Nevertheless, for these oxides, the oxide component after treatment is still important. We are currently working on improving the efficiency of the cleaning processes.

Nonetheless, it should be noted that a queue time ranging from $30 \mathrm{~min}$ to 2 hours is systematically observed between sample preparation and their surface characterisation. Then a regrowth of III-V native oxides could have occurred on treated samples and slightly influence the results. We are working on an experimental protocol to limit the air exposure between sample treatment and subsequent analyses.

Depending on the plasma nature, tendencies can be observed thanks to XPS analyses. Both plasmas seems to be efficient for eliminating As oxides whereas $\mathrm{Ga}$ and In oxides are more impacted by Ar-based plasma. Further experiments are in progress to confirm these tendencies.

\section{Conclusion - Perspectives}

We have demonstrated that a $300 \mathrm{~mm}$ platform usually dedicated for processing silicon wafers can be used for the in situ passivation/cleaning of III-V materials. The first results obtained with the employment of physical plasmas and described in this work are encouraging. We have found efficient processes for removing totally or partially III-V native oxides (depending on their nature). Moreover, we have observed first tendencies using different plasma varieties which will be useful in order to improve the efficiency of passivation/cleaning processes.

Impact of treatments on surface in terms of defects and roughness are under investigation (Atomic Force Microscopy 

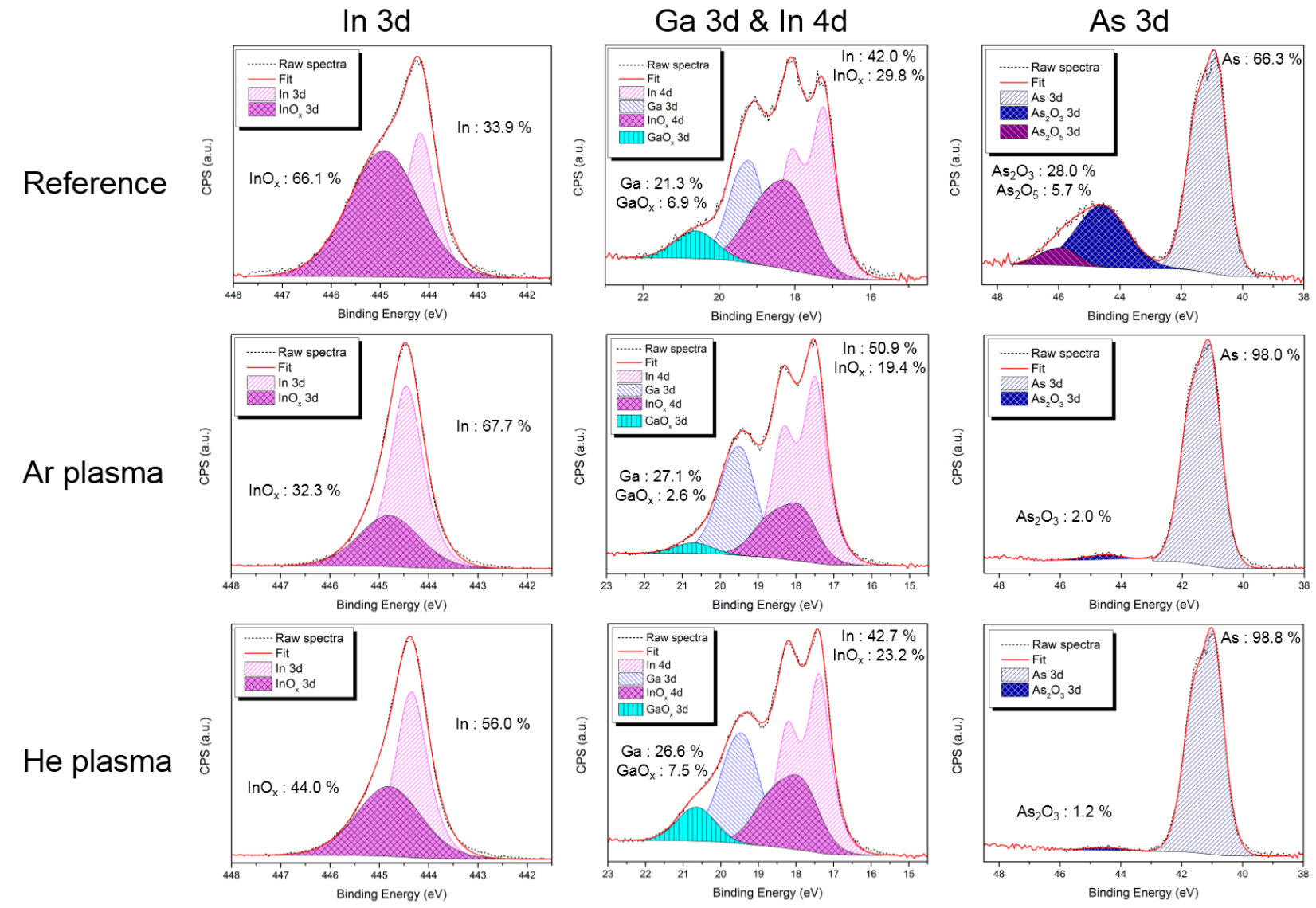

Fig. 3. XPS spectra peak fits of As $3 d$, In $3 d$ and Ga 3d, In $4 d$ regions for reference sample (top), Ar-based plasma treated sample (middle) and He-based plasma treated sample (bottom). The relative proportion of each component is indicated.

characterisations). A complete passivation/cleaning technology involving wet cleaning followed by in situ treatment is also being studied.

The processes developed in this study will be applied for the realisation of Ni-based self-aligned contacts on InGaAs and their impact in terms of electrical properties is planned to be investigated.

\section{ACKNOWLEDGEMENTS}

The authors would like to thank Thierry Baron and his team (CNRS-LTM, CEA-LETI) for the realisation of InGaAs epitaxy. This work has been partially supported by the French "Recherches Technologiques de Base" (Basis Technological Research) and RENATECH programs.

This project was supported by the public grant ANR-13NANO-0001 MOSINAS and the Equipex FDSOI11 projects.

\section{REFERENCES}

[1] J. R. Dale and R. G. Turner, "Simple ohmic contacts on gallium arsenide," Solid-State Electron., vol. 6, no. 4, pp. 388 - 389, 1963.

[2] S. Takagi, T. Iisawa, T. Tezuka, T. Numata, S. Nakaharai, N. Hirashita, Y. Moriyama, K. Usuda, E. Toyoda, S. Dissanayake, M. Shichijo, R. Nakane, S. Sugahara, M. Takenaka, and N. Sugiyama, "Carriertransport-enhanced channel CMOS for improved power consumption and performance," IEEE Trans. Electron Devices, vol. 55, no. 1, pp. 21-39, Jan 2008.
[3] Y.-C. Yeo, H.-C. Chin, X. Gong, H. Guo, and X. Zhang, "III-V MOSFETs: Surface Passivation, Source/Drain and Channel Strain Engineering, Self-Aligned Contact Metallization," ECS Trans., vol. 35, no. 3 , pp. 351-361, 2011.

[4] S. Takagi and M. Takenaka, "Prospective and Critical Issues of III-V/Ge CMOS on Si Platform," ECS Trans., vol. 35, no. 3, pp. 279-298, 2011.

[5] X. Zhang, H. Guo, X. Gong, Q. Zhou, Y.-R. Lin, H.-Y. Lin, C.-H. Ko, C. H. Wann, and Y.-C. Yeo, "In ${ }_{0.7} \mathrm{Ga}_{0.3}$ As Channel n-MOSFET with Self-Aligned Ni-InGaAs Source and Drain," Electrochem. Solid-State Lett., vol. 14, no. 2, pp. H60-H62, 2011.

[6] Ivana, E. Y.-J. Kong, S. Subramanian, Q. Zhou, J. Pan, and Y.-C Yeo, "CoInGaAs as a novel self-aligned metallic source/drain material for implant-less $\mathrm{In}_{0.53} \mathrm{Ga}_{0.47}$ As n-MOSFETs," Solid-State Electron., vol. 78, pp. 62-67, 2012.

[7] M. Rei Vilar, J. El Beghdadi, F. Debontridder, R. Artzi, R. Naaman, A. M. Ferraria, and A. M. Botelho do Rego, "Characterization of wetetched GaAs (100) surfaces," Surf. Interface Anal., vol. 37, no. 8, pp. 673-682, 2005.

[8] N. Liu and T. F. Kuech, "Interfacial chemistry of InP/GaAs bonded pairs," J. Electron. Mater., vol. 36, no. 3, pp. 179-190, 2007. 